\title{
Wavelet Transform - A New Tool for Analysis of Harmonics in Power Systems
}

\author{
D.S. Osipov, V.N. Gorunov, A.A. Bubenchikov and P.V. Katerov \\ Omsk State Technical University, Omsk, Russi
}

\begin{abstract}
This paper presents a review of application of discrete wavelet transform in power system transient analyses. Based on the discrete time domain approximation, the system components such as resistor and inductor are modeled respectively in discrete wavelet domain for the purpose of transient and steady state analyses. Numerical results for transient inductor model can be implemented by any kind of power system including normal and emergency operating modes.
\end{abstract}

\section{Introduction}

A wavelet transform (WT) is a mathematical tool for analyzing signals. Wavelet analysis is well suited to nonperiodic signals that may contain both stationary components and transient signals, such as the ones that can be found in power quality disturbances. The capacity of wavelets to adapt the window size improves the analysis of these types of nonstationary signals. Originally introduced in a group-theoretic setting, WT was soon realized to have powerful applications in various fields, which include analysing electric power systems [1], transient and steady state study electrical circuits [2] or analyzing power quality [3]. Harmonic, interharmonic, and subharmonic distortion can be studied using different WTs, in both stationary and nonstationary conditions, showing better performance in the measurement of time-varying waveform distortion [4].

The advantage of WT is that the band of analysis can be adjusted so that high frequency components and low frequency components can be detected precisely. Results from the wavelet transform are shown on both the time domain and the frequency domain.

Reference [4] provides extensive bibliographic review of applications of WTs in the measurement and analysis of harmonic distortion in power systems, discussing the performance of the different methods proposed in the technical literature.

\section{Wavelet theory}

Basically WT decomposes a given function $f$ into its components on different scales or frequency bands. This is done by convoluting $f$ with the translated and dilated wavelet $\psi$ :

$$
F_{\psi}(b, a)=\frac{1}{\sqrt{a}} f(t) \psi\left(\frac{t-b}{a}\right) d t
$$

In Equation (1) $f(t)$ is the signal to be analyzed, $F_{\psi}$ is the wavelet transform of $f(t)$ based on mother wavelet $\psi(t)$, and $b$ and $a$ are the shift and dilation parameters, respectively. Each wavelet is created by scaling and translation operations in a special function called mother wavelet. A mother wavelet is a function that oscillates, has finite energy and zero mean value. In timefrequency domain the information regarding both time and frequency are preserved making it better than Fourier transform, i.e. a frequency domain approach. Also, the problem of fixed window size as in short time Fourier transform (STFT) is solved in WT.

A wavelet transform can be divided into a continuous wavelet transform (CWT) and a discrete wavelet transform (DWT). Fig. 1 represents CWT of the nonstationary signal under examination, the CWT giving a clear idea of amplitude, frequency and time variation in three-dimensional space.

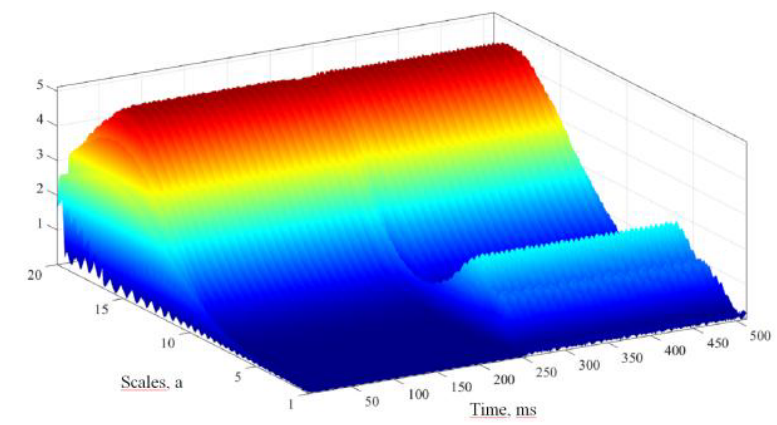

Figure 1. Continuous wavelet transform of nonstationary current signal under examination 
The calculation of wavelet spectrum under CWT causes substantial computational difficulties at a continuous variation of parameters $\mathrm{j}$ and $\mathrm{k}$.

The set of functions $\psi \mathrm{j}, \mathrm{k}$ is redundant, therefore to preserve a possibility of desampling out of its conversion, the sampling of these parameters is necessary. Sampling is often calculated by powers of two.

For the time being Haar wavelets have been replaced by other types of wavelets, but still Haar wavelets are rather comfortable to form theoretical basis of signals examination using wavelet transforms.

For the purpose of numerical analysis, only DWT is briefly introduced here. More detailed information on wavelet theory is available in [5], [6].

\section{Transient inductor model}

Since we know that the inductor current cannot change its value instantaneously, as otherwise the inductor voltage $v_{L}=L \cdot d i_{L} / d t$ would become infinite, it follows that the inductor current immediately after closing the switch, must be the same as the inductor current just before closing the switch:

$$
i_{L}\left(0_{-}\right)=i_{L}\left(0_{+}\right)
$$

Consider the RL circuit of Fig. 2 with $R=6.35 \Omega$, $L=0.127 H \quad$, and $v(t)=\sqrt{2} \cdot 220 \sin (2 \pi f+\varphi) \quad(\mathrm{AC}$ voltage source $\left.f=50 \mathrm{~Hz}, \varphi=30^{\circ}\right)$. We may find the expressions for the inductor current $i_{L}(t)$ for $t>0$, and plot them. Determination of the optimal parameters of RLC models is carried out by processing the instantaneous values of non-sinusoidal voltage and current signals of electric devices measured in the frequency range, limited with conditions of the used model adequately [7].

Using Kirchhoff's voltage law around the loop (Fig. 2) in the clockwise direction, we have ordinary differential equation:

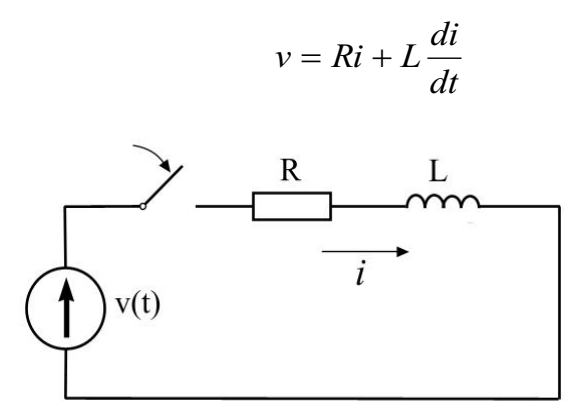

Figure 2. Analyzed RL-circuit transient

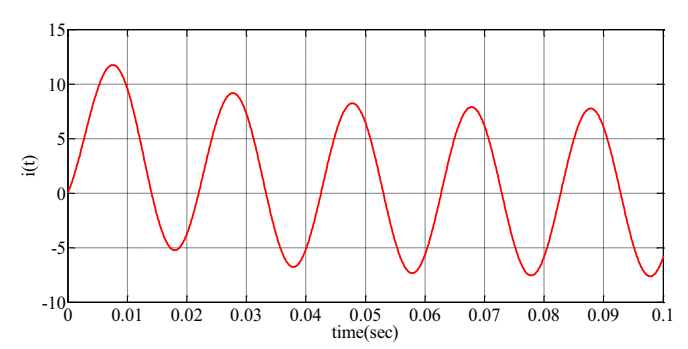

Figure 3. Inductor current
The solution is given by:

$$
i_{L}(t)=7.68 \sin \left(2 \pi f-51^{0}\right)+5.97 e^{-t / 0.02}
$$

Reference [7] proposes to solve linear ordinary differential equations with wavelets - the wavelet recursion method.

Let $v(t)$ and $i_{L}(t)$ represent, respectively, the response and the forcing function vectors denoted at time $t=n \Delta t$, where $\Delta t$ is the sampling interval. We may assume that the DWT representations of the vectors are:

$$
\begin{aligned}
& v(t)=k \sum_{j} \sum_{k} v_{j, k} \psi_{j, k}(t) \\
& i_{L}(t)=k \sum_{j} \sum_{k} i_{j, k} \psi_{j, k}(t)
\end{aligned}
$$

The Right Formula Backward Difference (RFBD) equations begin with the backward difference approximation of the derivative in discrete time as follows [8]:

$$
\frac{d i_{L}}{d t}=\frac{i_{L}(t)-i_{L}(t-\Delta t)}{\Delta t}
$$

Given the equation (6), the equation (3) can be rewritten:

$$
\frac{1}{\Delta t}\left[i_{L}(t)-i_{L}(t-\Delta t)\right]+\frac{R}{L} i_{L}(t)=\frac{1}{L} v(t)
$$

When the discrete wavelet series for $v(t)$ and $i_{L}(t)$ are substituted into a scalar version of Equation (7) using Equations (4-5) for the derivative approximation, one obtains (for small $\Delta t$ )

$$
\begin{aligned}
& \frac{1}{\Delta t}\left[k \sum_{j} \sum_{k} i_{L j k} \psi_{j k}(t)-k \sum_{j} \sum_{k} i_{L j k} \psi_{j k}(t-\Delta t)\right]+ \\
& +\frac{R}{L}\left[k \sum_{j} \sum_{k} i_{L j k} \psi_{j k}(t)\right]=\frac{1}{L}\left[k \sum_{j} \sum_{k} v_{j k} \psi_{j k}(t)\right]
\end{aligned}
$$

where

$$
\psi_{j k}(t)=\frac{1}{\sqrt{a_{0}}} \psi\left(\frac{t}{a}-b_{0} k\right)
$$

and

$$
\psi_{j k}(t-\Delta t)=\frac{1}{\sqrt{a_{0}}} \psi\left(\frac{t-\Delta t}{a}-b_{0} k\right)
$$

The discrete wavelet transform has a fast recursive form that is most easily implemented using bank theory [9]. This algorithm requires only $O(N)$ steps instead of $O(N \cdot \ln (N)$ steps required by the FFT. Fig. 4 shows a simplistic way of looking at a wavelet decomposition of signal. 


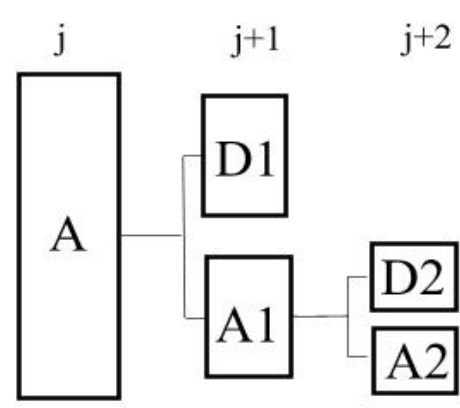

Figure 4. Simplistic view of a wavelet decomposition

Wavelet transform algorithm produces DWT coefficients starting from separating the original signals $v(t)$ and $i_{L}(t)$ of length $\mathrm{N}$ (number of simples) to 2 set of coefficients: approximate coefficients A1 by low pass filter and detail coefficients D1 by high pass filter. The length of each filter is equal to half of original length by down sampling function. The next step splits the approximate coefficients A1 in two parts again by the same process but replaces by $\mathrm{A} 1$ and produces $\mathrm{A} 2$ and D2 and so on. Thus, if the decomposition stopped at this point, the approximation of the signal at level $j$ could be reconstructed by the sum of the approximation at level $j+1$ and the details at level $j+1$. That is

$$
A_{j}=A_{j+1}+D_{j+1}
$$

Since the wavelet and scaling functions have the aforementioned properties of orthonormality, one may view the Equation (8) as a linear combination of the detail coefficients and the approximation coefficients.

In the Multiresolution analysis (MRA) theory described by Mallat [5], a wavelet expansion of function (in this case function of inductor current and voltage) will have the form:

$$
\begin{aligned}
& i_{L}(t)=\sum_{k=-\infty}^{\infty} i_{L j, k}^{A} \varphi_{j, k}(t)+\sum_{j=J}^{\infty} \sum_{k=-\infty}^{\infty} i_{L j, k}^{D} \psi_{j, k}(t) \\
& v(t)=\sum_{k=-\infty}^{\infty} v_{j, k}^{A} \varphi_{j, k}(t)+\sum_{j=J}^{\infty} \sum_{k=-\infty}^{\infty} v_{j, k}^{D} \psi_{j, k}(t)
\end{aligned}
$$

In equation (12-13) the superscripts $\mathrm{D}$ and $\mathrm{A}$ represent the detail and approximation coefficients.

Based on Parseval equality one can calculate the effective current within the required range of frequencies. In this paper the effective elementary frequency $(50 \mathrm{~Hz})$ can be obtained by approximation and detailed wavelet coefficients of the third level decomposition (in this case a maximum one):

$$
I_{(50)}=N^{-\frac{1}{2}} \sqrt{\sum_{k=0}^{k_{m}}\left(i_{3, k}^{A}\right)^{2}+\sum_{k=0}^{k_{m}}\left(i_{3, k}^{D}\right)^{2}}
$$

The effective currents of higher harmonics $I_{(v)}$ are defined by detailed coefficients of the first two decomposition levels:

$$
I_{(v)}=N^{-\frac{1}{2}} \sqrt{\sum_{j=1}^{2} \sum_{k=0}^{k_{m}}\left(i_{j, k}^{D}\right)^{2}}
$$

where, $N$ - the number of readings of the discrete signal examined

Thus the effective values obtained can be substituted in the formula of waveform distortion factor:

$$
K_{u}=\frac{I_{(v)}}{I_{(50)}}=\sqrt{\frac{\sum_{j=1}^{2} \sum_{k=0}^{k_{m}}\left(i_{j, k}^{D}\right)^{2}}{\sum_{k=0}^{k_{m}}\left(i_{3, k}^{A}\right)^{2}+\sum_{k=0}^{k_{m}}\left(i_{3, k}^{D}\right)^{2}}}
$$

The task of wavelet analysis implementation for the voltage and current sine - wave signals distortion identification in industrial power systems with the wavelet carrier choice being substantiated is solved in [5]. The author examines the applicability of more than ten types of wavelets in power quality indices determination tasks. The four types of wavelets are presented in this paper (Table 1). With the wavelets specified we may define the effective currents of elementary frequency and higher harmonics with the subsequent waveform distortion factor being determined.

Table 1. Comparison of calculation accuracy of some wavelets

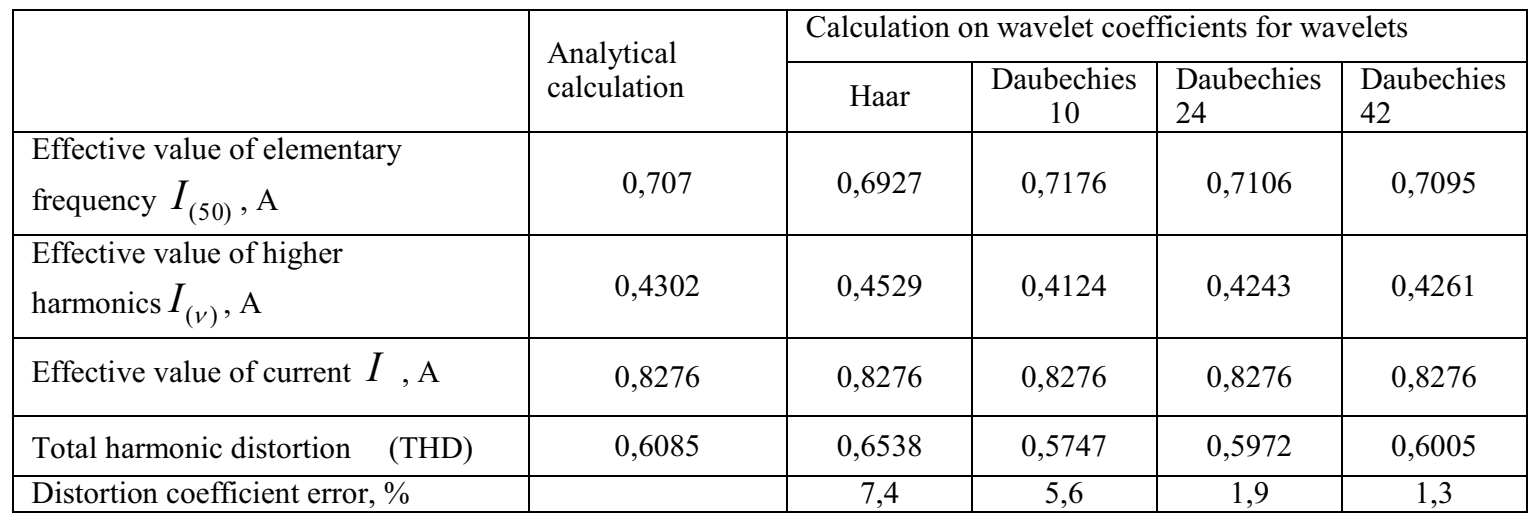

Table 1 illustrates that the type of the wavelet used defines the distortion coefficient calculation accuracy.
Daubechies wavelets of orders 24 and 42 (db24, db42), demonstrate the most acceptable results, the error not 
exceeding $2 \%$. The proposed techniques of defining harmonic composition of current or voltage and the modern methods of active filtering of higher harmonics [6], [8] may help solve the tasks of power quality using the advantages of wavelet transform.

The decomposition of the signal into different frequency bands is simply obtained by successive high pass and low pass filtering of the time domain signal. The original signal $v(t)$ (or $\left.i_{L}(t)\right)$ is first passed through a half-band high pass filter $\mathrm{g}[\mathrm{n}]$ and a half-band low pass filter $\mathrm{h}[\mathrm{n}]$. The half-band low pass filter removes all frequencies that are above half of the highest frequency, while the half-band high pass filter removes all frequencies that are below half of the highest frequency of the signal. The low pass filtering halves the resolution, but leaves the scale unchanged. The signal is then subsampled by two since half of the number of samples is redundant, according to the Nyquist's rule [10]. There are many perfect reconstruction filters. We may notice that Haar filter is the first Daubechies filter.

\section{Nunerical experiment results}

In this section a simple differential equation (3) is solved using proposed in [8] Right Formula Backward Difference method.

\subsection{Steps of DWT analysis procedure}

Step 1. Chose the type of wavelet. One reason for the popularity of wavelet technique is the vast choices of wavelets as the basis function, compared to the fixed choice of sine and cosine functions for Fourier analysis. This enables wavelet analysis to be adapted according to the expected characteristics of the signal. A coarse (irregular) wavelet is more suitable for a signal with sharp or abrupt transitions, while those smooth ones are better analyzed using regular wavelets. A mother wavelet is chosen by the user for the analysis. The selection of the mother wavelet is not a trivial issue. Different mother wavelets have different attributes which may or may not benefit in the analysis. Therefore the selection of the appropriate mother wavelet may be crucial [8]. We shall take Haar wavelet for the numerical experiment, which can be described as:

$$
\psi(t)= \begin{cases}1 & 0 \leq t \leq 1 / 2 \\ -1 & 1 / 2 \leq t \leq 1 \\ 0 & \text { otherwise }\end{cases}
$$

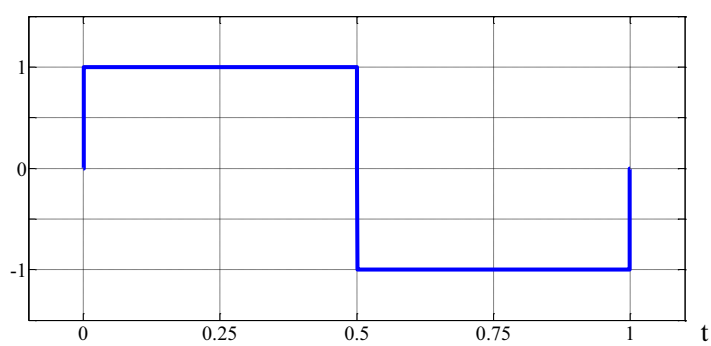

Figure 5. Haar wavelet
Its scaling function $\varphi(t)$ can be described as:

$$
\varphi(t)=\left\{\begin{array}{cc}
1 & 0 \leq t \leq 1 \\
0 & \text { otherwise }
\end{array}\right.
$$

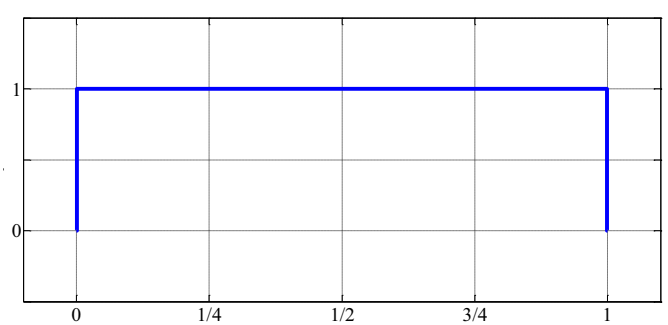

Figure 6. Haar scaling function

Step 2. Forward DWT with the selected wavelet filter, MRA decomposition scheme is performed on the system input signals to get their wavelet transform coefficients (Equations 4-5).

Step 3. Solve system equivalent circuit in wavelet domain.

$$
\begin{gathered}
\frac{1}{a_{0}^{j} \Delta t} i_{L j k}^{A}-\frac{1}{a_{0}^{j} \Delta t} i_{L j k-1}^{A}+\frac{R}{L} i_{L j k}^{A}=\frac{1}{L} v_{j k}^{A} \\
\frac{1}{a_{0}^{j} \Delta t} i_{L j k}^{D}-\frac{1}{a_{0}^{j} \Delta t} i_{L j k-1}^{D}+\frac{R}{L} i_{L j k}^{D}=\frac{1}{L} v_{j k}^{D}
\end{gathered}
$$

In this numerical experiment $j=1$, and so $a_{0}^{j}=2^{1}=2 \quad, \quad$ The choosen step time $\Delta t=10^{-4}(s)=100 \mu s$.

$$
\begin{gathered}
i_{L j k}^{A}=\frac{\left(v_{j k}^{A} / L\right)+5 \cdot 10^{3} \cdot i_{L j k}^{A}}{5 \cdot 10^{3}+(R / L)} \\
i_{L j k}^{D}=\frac{\left(v_{j k}^{D} / L\right)+5 \cdot 10^{3} \cdot i_{L j k}^{D}}{5 \cdot 10^{3}+(R / L)}
\end{gathered}
$$

Step 4. Time-frequency response analysis: with the equivalent circuit, system transfer function in wavelet domain can also be set up. thus the characteristic of system time-frequency response can be easily achieved.

Step 5. Inverse DWT The MRA reconstruction scheme is performed on the wavelet transform coefficients of output signal to obtain their waveform in discrete time domain.

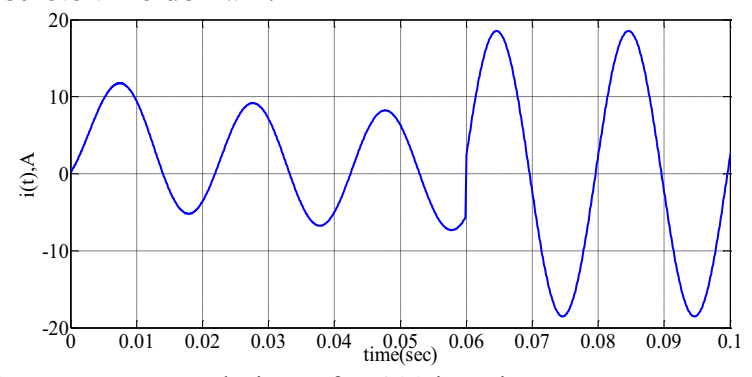

Figure 7. RFBD solutions after 300 iterations 


\subsection{Example analysis}

Fig. 7, Fig. 8 illustrate how the recursion progress toward the solution (solution after 300 and 600 iterations).

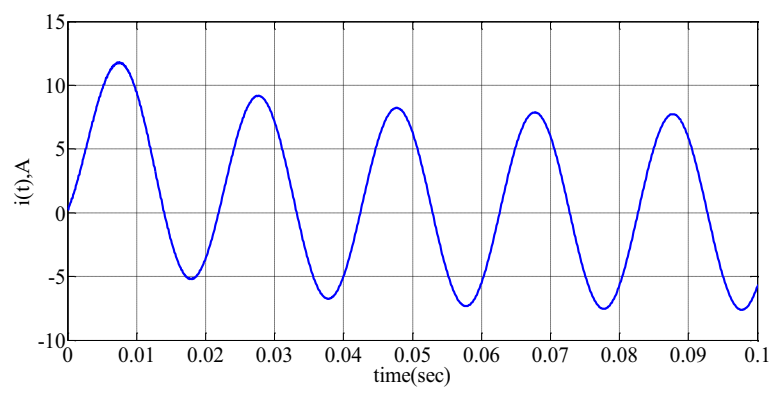

Figure 8. RFBD solutions after 600 iterations

\section{Conclusion}

This paper has presented a method for transient analysis of electric power systems. This method, referred to as the wavelet recursion method, makes use of a relatively new mathematical tool, the wavelet transform, which has certain benefits over techniques such as Fourier analysis when analyzing transient and nonstationary signals. Through the application of operator representation theory, the system component models in the wavelet domain are set up. Proposed in [2,8] models can be implemented by any type of orthogonal wavelet transform. Once the discrete time domain models are obtained, wavelet domain models can be easily calculated by MRA decomposition scheme.

An assessment of the advantages and disadvantages of the wavelet recursion method are as follows [8]:

- The DWT produces sparse representations of signals, thus sparse matrix techniques could be applied to this method to improve speed.

- In many cases, the wavelet recursion method converges relatively fast.

- Wavelet analysis software is readily available and adaptable to a variety of applications, including power engineering.

- Matrix inversions (at most, one inversion per level of decomposition) may be required.

In this paper the resistor model is considered as a scalar, the inductor model is based on the differential operator. The proposed scheme is suitable for transients and time varying analysis. Along with the proposed procedure, a switching of inductor is used as an example in this paper.
The paper was prepared in Omsk Srate Technical University with financial support from Ministry of Education and Science in the framework of the agreement № 14.577.21.0097 on August 22, 2014. A unique identifier of applied research RFMEF157714X 0097.

\section{References}

1. P. F. Ribeiro, "Wavelets: A new approach to analyze power system distortions" in Proc. EPRI-Power Quality Steering Committee, Baltimore, MD, (1993).

2. T. Zheng, E. Makram, and A. A. Girgis, "Power system transient and harmonic studies using wavelet transform," IEEE Trans. Power Del., 14, no. 4, pp. 1461-1468, Oct. (1999)

3. S. Santoso, E.J. Powers, and W.M. Grady, "Wavelet-based Power Quality Event Identification System, Part 1The Wavelet Transform and Feature Extraction of Power Quality Disturbances and Part 2: Design and Implementation," ERPI Report, August (1997)

4. J. Barros, R. I. Diego and M. de Apraiz. "Applications of Wavelet Transform for Analysis of Harmonic Distortion in Power Systems: A Review". IEEE Transactions on instrumentation and measurement, 61, no. 10. pp. 2604-2611, October (2012)

5. Mallat, S. "A wavelet tour of signal processing" Academic Press, New York, (1998)

6. Dwight F. Mix and Kraig J. Olejniczak "Elements of wavelets for engineers and scientists". John Wiley \& Sons, Inc. New York, NY, USA (2003)

7. A.N. Kirichenko, V.N. Gorunov, A.V. Schekochikhin, V.V. Barskov. "Algorithms of optimal parametric synthesis of RLC models in the problem of parameter identification of electrical devices". Dynamics of Systems, Mechanisms and Machines (Dynamics), (2014)

8. A. W. Calli, "Analysis of Electrical Transients In Power System Via A Novel Wavelet Recursion Method", Ph.D. Dissertation, Purdue University (1997)

9. G. Strang and T. Nguyen. Wavelets and Filter Banks. Wellesly-Cambridge Press. Wellesly. MA. (1996).

10. I.D. Nicolae, P.M. Nicolae , M.S. Nicolae. "Realtime Analysis Using Discrete Wavelet Transform in Power Systems" 15th International Power Electronics and Motion Control Conference, EPEPEMC 2012 ECCE Europe, Novi Sad, Serbia 